\title{
España, una meditación política: Cataluña y Euskadi
}

\author{
Spain, a political meditation: Catalonia and Euskadi
}

Mikel Aramburu Zudaire

Universidad de Navarra

Resumen: El artículo expone las principales ideas del filósofo español José Luis L. Aranguren (1909-96), a partir de un libro que publicó en 1983, sobre la situación política de España durante los primeros años de la última Transición democrática tras la muerte del dictador Franco (1975). En particular se hace referencia a lo relativo a los territorios de Cataluña y Euskadi (Comunidad Autónoma Vasca), donde se pueden apreciar continuidades y diferencias con la situación actual además de sobresalir la adelantada visión y vigencia de gran parte del análisis y reflexión arangurenianos.

Palabras clave: España, Cataluña, Euskadi (Comunidad Autónoma Vasca), Nacionalismo, Democracia.

\begin{abstract}
The article presents the main ideas of the Spanish philosopher José Luis L. Aranguren (1909-96), from a book published in 1983 on the political situation of Spain during the first years of the last democratic transition (following the death of dictator Franco in 1975). In particular, reference is made to the territories of Catalonia and Euskadi (Spanish Basque Country), where we can see continuities and differences with the current situation as well as to appreciate the advanced vision and validity of much of the analysis and reflection made by Aranguren.
\end{abstract}

Key words: Spain, Catalonia, Euskadi (Spanish Basque Country), Nationalism, Democracy.

Recibido: 15-10-17. Aceptado: 3-4-18

Mikel Aramburu Zudaire es Doctor en Historia y Master en Filosofía, y trabaja como Profesor de Filosofía en Enseñanza Secundaria del Gobierno de Navarra (España).

Contacto: Paseo José Saramago, 13-3B, 31160, Orkoien (Navarra, España) aranzumiel@gmail.com

Cómo citar: Aramburu, M. (2018). España, una meditación política: Cataluña y Euskadi. Revista Stultifera, 1 (1), 45-59. ISSN 0719-983X

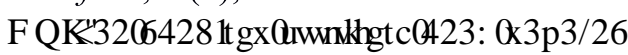




\section{ESPAÑA, UNA MEDITACIÓN POLÍTICA: CATALUÑA Y EUSKADI}

España se hunde (...)

El acuerdo en lo cotidiano

no existe;

tampoco la voluntad de vivir en común,

ni el plebiscito de "tous les jours", principios que se conjugan en pasado.

Eso es todo,

esta nación se hunde y se disgrega

"tempus fugit irreparabile"

(López de Cerain, 2016, p.77)

En 1983, el filósofo español José Luis L. Aranguren (1909-96), nacido en Ávila pero con raíces vascas, publicaba un libro con el título que encabeza este artículo, una meditación del autor sobre la entonces recién recuperada democracia en España (1995). El libro es una ordenada y refundida compilación de artículos aparecidos entre 1979 y 1982 en el periódico El País, además de dos conferencias, una no impresa, y un artículo en la revista Tiempo. Se presentan en seis bloques o capítulos y en nuestro trabajo abordaremos en concreto los titulados: "Cataluña y España" y "Liturgia y apocalipsis en Euskadi”. Creo resulta de interés volver a la reflexión de este pensador, aún válida para el tiempo actual, pues se echan en falta voces y plumas de la categoría humana e intelectual de Aranguren que iluminen esta hora de incertidumbres. Quisiera destacar el valor de los citados capítulos que merecieron una atención específica del autor sobre aquellos territorios, pues resulta muy oportuno recordarlos en este momento histórico de España. Sin duda las propias palabras del profesor abulense son en sí mismas, con su particular, rico y creativo estilo literario, lo suficientemente elocuentes y cada lector o lectora puede sacar las conclusiones que estime oportunas. A mí solo me gustaría apuntar lo que aún me parece vigente e incluso resulta profético o visionario, escrito hace más de 30 años, junto a otras cuestiones más discutibles o que han quedado superadas por la marcha de la historia.

Aranguren mismo —en el prólogo al volumen de sus Obras Completas que recoge el libro en cuestión (1995) - reconocía la actualidad del mismo, doce años después, aunque el apartado sobre el País Vasco le resultaba ya demasiado largo y, en su final, no acertado (sic). Lo que sí sigue siendo muy necesario, desgraciadamente después de tanto tiempo - y de ello escribe en el primer capítulo del libro que nos sirve de contexto-, es recuperar e insistir en un concepto-clave de su pensamiento, a saber, la democracia como moral, pues no es algo natural sino racional y 


\section{MIKEL ARAMBURU ZUDAIRE}

cultural, moral, que, sin dejar de ser realista y eficaz, consiste siempre en un largo y difícil proceso de democratización que no termina nunca y exige la participación de toda la ciudadanía desde una cultura política viva (democracia participatoria, en palabras de Aranguren, y no meramente delegada). Creo que es, tal vez, la mejor vía para lograr la regeneración —él alude a un nuevo regeneracionismo - de todo el sistema democrático de nuestro tiempo, corrompido de raíz, y prevenir su tendencia a la degradación, dada la frágil condición humana, tal como ya lo previeron desde sus orígenes en la antigua Grecia (como se aprecia en la Política de Aristóteles).

Por otro lado, si ya al comenzar los años ochenta se había dado un cambio importante en la sociedad española respecto a la de los años treinta, también ahora podemos percibir otro gran cambio social bien entrados en el siglo XXI. Ciertamente lo del centrismo como opción socioelectoral mayoritaria parece continuar siendo decisivo para ganar las elecciones y es lo que dio el triunfo en 1982 al Partido Socialista Obrero Español (PSOE) y después al turnismo PSOEPartido Popular (PP) hasta hoy mismo. Llama la atención el término utilizado por Aranguren de transideología, que no lo desarrolla en el libro, pero que visto desde el presente recoge bien la crisis de las ideologías clásicas de la edad contemporánea ya clara en aquella época y acentuada en las décadas siguientes de dominio del posmodernismo, con la caída del muro de Berlín y el fin de la historia (en conocida expresión de Fukuyama). Además, tras la última crisis sistémica que hemos vivido en estos años en España, no solo económica y financiera, y de la que se está anunciando su salida en datos macro y no tanto en hechos concretos para amplias capas sociales, no se puede decir que estemos ahora en un tiempo político de esperanza y menos de entusiasmo -como Aranguren piensa que se estaba viviendo aquella victoria del PSOE en el 82- sino que más bien la población asiste entre indignada o decepcionada y escéptica o hasta cínica al descrédito de la actividad política y de quienes la ejercen en su nombre.

En un segundo capítulo trata de la monarquía y su relación con la democracia y nos interesa, como he señalado para el primer capítulo, de marco contextual que revela sus ideas en aquella época. Es evidente que, tras el intento fallido de golpe de Estado de 1981, al menos la figura del rey demócrata había salido reforzada (Juan Carlos I), según reconoce en el libro, y era difícil plantearse un cambio de régimen. Comparto con Aranguren que en caso de darse ese cambio -al que él no se cierra y ve posible más pronto o más tarde (pienso hoy más cercano dado el descrédito general de la institución a pesar de la mejor imagen del nuevo rey Felipe VI)—, no consistirá en 


\section{ESPAÑA, UNA MEDITACIÓN POLÍTICA: CATALUÑA Y EUSKADI}

la mera recuperación de la República de 1931 sino en otra república, sin nostalgia. Pero está claro que lo que Aranguren consideró un episodio definitivamente clausurado de la historia de España -el de la República, la guerra civil y la larga dictadura del general Franco-, no lo estaba aún como lo demuestra el clamor memorialista de los últimos tiempos, no concluido todavía, para reparar y hacer justicia a tantas víctimas no reconocidas de aquel conflicto bélico y posterior represión.

Empezando ya por lo expuesto sobre Cataluña y España, hay que leerlo desde la comprensión y admiración de Aranguren por la cultura y forma de vida catalana, pues para él ir a Barcelona era una fiesta donde se sentía bien acogido y recibía mayor cariño que en ninguna otra parte. Pero, en aquellos años 1980, lo que percibe es el desencanto de Cataluña y éste se correspondía con el gobierno del antiprestidigitador presidente Jordi Pujol, vuelto "al seny y la rauxa de la típica burguesía catalana" (Aranguren, 1995, p. 666). Para superar dicho desencanto - que hoy resulta casi irreconciliable desencuentro y desconexión - Aranguren veía necesaria la autocrítica por ambas partes. Una parte para entender y asumir que históricamente España nunca ha sido una nación sino una monarquía asentada sobre nacionalidades diversas, ni tampoco un estado sino más bien un imperio. Y la otra parte para aceptar que su ser nacional y sobre todo su lengua no eran compartidas por un sector importante de la población catalana, especialmente la originaria de la masiva inmigración de toda la Península Ibérica a lo largo de la segunda mitad del siglo XX. Además, se preguntaba entonces nuestro autor si la lengua catalana no estaba sufriendo "un cierto estancamiento literario y (...) un cierto estancamiento de su dinamismo y fuerza de invención coloquial" (Aranguren, 1995, p. 667)..

La primera autocrítica la considero muy razonable y, tras décadas de Estado de las Autonomías, parece que debería estar asumida la plurinacionalidad de España por gran parte de la ciudadanía española y la mayoría de los partidos políticos estatales, pero aún enciende vivos debates y controversia, sobre todo mediática. Y la segunda autocrítica resulta más complicada de valorar pues, si nos centramos en la lengua, creo que goza de buena salud y su uso está bien extendido y normalizado (otra cuestión es la calidad). Además, ha existido una suficiente — para bastantes no la suficiente- mayoría social y política partidaria de, al menos, celebrar el referéndum de independencia del pasado primero de octubre y en ello no hay diferencias por el origen o nacimiento. 


\section{MIKEL ARAMBURU ZUDAIRE}

Volviendo al idioma catalán, Aranguren hace un alegato a favor de toda lengua, por minoritaria que sea, como "genuina e irreductible perspectiva sobre la realidad y, todavía más, creadora de realidad. La lengua tiene que ser defendida y liberada, sí, pero lejos de todo proteccionismo conservacionista, ha de ser también ilustrada creadoramente por los propios catalanohablantes" (Aranguren, 1995, p. 671). No obvia el problema político de disponer o no de un estado para defender mejor el idioma, pero su papel, que él quiere encarnar, es el de intelectual para que el Estado se organice, ampare la libertad y fomente la cultura.

Aranguren apuesta, pues, por unas bases más culturales que políticas para repensar la nacionalidad catalana, para inventar culturalmente una nueva forma de existencia colectiva que bien podría cerrarse sobre sí misma o bien abrirse a España y a Europa. Fiel a su genuino pensamiento, anima a admitir también la heterodoxia dentro del catalanismo para que haya una reflexión crítica sobre él y, con todo ello, formula su propuesta de la necesidad de una nueva catalanidad que envuelva en su proyecto futuro a unos y otros catalanes. Esto suena profético desde el momento presente por llamar al entendimiento mutuo. No sé si ahora mismo será posible llegar a él y en qué términos que satisfagan a todas las partes, pero opino, como él, que sería lo más conveniente para la convivencia social.

Aranguren insiste asimismo en el diálogo castellano-catalán, intelectual y de intelectuales, ya dado en la historia reciente, que aborde también la dimensión política para alcanzar una síntesis entre el reconocimiento pleno de la cultura catalana y las formulaciones correspondientes en dicho plano político. Por eso cree que, además del Estado, es también tarea de los intelectuales, de los españoles y de los catalanes interculturalmente. La intelectualidad castellana, al menos la de su edad, debe mucho a Cataluña, a su modo de vivir y sentir la vida, no de manera tan trágicamente unamuniana como la castellana, sino más sensible y libre, e incluso a la forma de luchar por su país tan diferente a la del pueblo vasco como se ve en el siguiente capítulo del libro.

Una reflexión final la dedica al citado Estado de las Autonomías, emanado de la Constitución española de 1978, que para él tiene sentido en comunidades con una realidad diferencial clara, histórica, geográfica, antropológica, cultural y económica, como lo es la de Cataluña y el País Vasco pero considera —en este caso pienso, equivocadamente- que en las últimas elecciones del 82, por los resultados, había habido una falta de emoción colectiva nacionalista y se tendría que conjugar la querida autonomía con el principio de solidaridad entre 


\section{ESPAÑA, UNA MEDITACIÓN POLÍTICA: CATALUÑA Y EUSKADI}

todos los españoles en favor del cambio general prometido por el partido triunfante (PSOE). Tal vez eso pareció a la vista de aquellos comicios, pero sabemos el papel que ha jugado, a lo largo de estas décadas y hasta ahora, el sentimiento nacionalista periférico, sobre todo vasco y catalán, y sus mediaciones políticas en los respectivos gobiernos autonómicos y en la misma gobernabilidad del Estado español, aunque no precisamente movidos por la solidaridad interterritorial.

Y pasando al capítulo referido a Euskadi, parece que también fue criticado por algunos, según confiesa el editor de las obras completas, al presentar una visión catastrofista sobre un País Vasco independiente y al decantarse hacia el realismo. Si lo he entendido bien, yo no comparto esta impresión, al contrario, me parece que Aranguren acierta con dicho realismo y es un adelantado en ciertos puntos de su reflexión. Esta la centra en dos grandes temas, uno el de la lengua propia, el hecho diferencial más ostensible, y otro el del terrorismo de la banda ETA. Al euskera o vascuence lo considera como uno de esos lenguajes litúrgicos (caso del latín) y reduplicativamente simbólico. Según Aranguren, salvo en caso de ser usado como habla rural o doméstica, de endogrupo, o para quienes lo estudian y aprenden arrastrados por un cuasirreligioso entusiasmo, "no es, hoy por hoy, una lengua efectiva sino solo potencialmente; pero es un «símbolo» catalizador, un ritual, un conjunto de fórmulas de comunión y, asimismo, un arma de combate para la «liberación»” (Aranguren, 1995, p. 678-79). Creo que, en cierto modo, así ha sido, en especial y desgraciadamente lo último que señala, pero en lo de su efectividad, con todas las limitaciones y carencias sobre el uso o la calidad, pienso se ha ido avanzando en las últimas décadas con la euskaldunización de las generaciones jóvenes y la normalización sociolingüística. En Navarra, de donde soy nacido y en donde resido, como parte histórica y cultural esencial de la tierra del vascuence o euskera (Vasconia o Euskal Herria), la situación y el proceso es diferente y demandaría una meditación específica.

Termina Aranguren este apartado sobre el lenguaje relacionándolo con el sentido de la vida y en el caso del euskera afirma - me parece positivamente por lo que acabo de decir de la normalización-que:

Si no se vuelve nostálgicamente arcádico - lo que es un peligro real de Euskadi- vive y, sobre todo, vivirá mirando adelante y pasando de ser nada menos, pero también nada más, que ritual, a convertirse en la forma de vida del pueblo vasco y de su creativa personalidad comunitaria. (1995, p.680) 


\section{MIKEL ARAMBURU ZUDAIRE}

En cuanto a lo que desarrolla sobre el terrorismo de ETA —además durante aquellos años de plomo de 1980 en que estaba muy activo- es sugerente, aunque hoy no resulte tan novedoso, el enmarque que le da situándolo dentro de una secularización de la violencia religiosa. Hace para ello primeramente un breve recorrido histórico a partir del franquismo tardío cuando el movimiento juvenil vasco se mostraba aguerrido, activista, apocalíptico, de lucha frontal contra el poder, mientras el del resto de España practicaba más la conspiración como forma de contestación al régimen. Entonces todo demócrata español estaba de parte de los vascos como sucedió en el conocido como juicio de Burgos (1970) o en el atentado contra el impopular presidente del Gobierno almirante Carrero Blanco (1973), aquel saltar por los aires técnicamente perfecto, y que se percibió como el reverso lamentable e inevitable de una operación política para yugular el continuismo del régimen franquista. Muchos lo agradecieron y se beneficiaron de él aunque después, en opinión de Aranguren, se habían rasgado hipócritamente las vestiduras ante los crímenes etarras (Aranguren, 1995, p. 681).

Siguiendo su relato histórico, nos cuenta que, con la llegada de la democracia, muchos jóvenes y menos jóvenes no la aceptaron como tal y a otros, como a él, les había dejado insatisfechos. Unos reaccionaron desde la crítica intelectual, otros desde la acracia o la mansa utopía y hasta el pasotismo, mientras en el caso vasco fue la lucha sin cuartel. Aranguren hace aquí una distinción muy atrevida en su tiempo e incluso ahora mismo: aunque la violencia de ETA es para él objetivamente terrorismo, no lo es desde el punto de vista de su interpretación subjetivocomunitaria. En realidad, ETA asumió la definición franquista hecha suya por el atropellado ministro del régimen Manuel Fraga (luego líder del PP) de que la guerra contra la anti-España (separatismo, comunismo) no terminó en 1939 y, por eso, los actos perpetrados por la banda armada, por mal que le parezca a Aranguren (y a mí también), eran didácticamente explicados al pueblo vasco como operaciones de guerra contra un país invasor y ocupante, además de ser un conflicto entre capitalismo y marxismo. Aranguren concluye, tomando la idea de otro profesor amigo y marxista, que se trataba de un conflicto entre el nacionalismo vasco y el nacionalismo español, y que la causa abertzale (patriota) era, inseparablemente, de guerra y revolución (socialista). Muchos jóvenes vascos habían rechazado la democracia de reforma sin ruptura, con sus reglas de juego, que sí estaban aceptando los catalanes (Aranguren, 1995, p. 681-82). Son curiosos los vaivenes de la historia y cómo han cambiado las tornas hoy día entre Euskadi y Cataluña. 


\section{ESPAÑA, UNA MEDITACIÓN POLÍTICA: CATALUÑA Y EUSKADI}

Respecto a la citada secularización de la violencia religiosa (bien analizada, según apunta Aranguren, por el historiador y filósofo francés René Girard, 2006), nuestro autor toma en consideración dos supuestos que me parecen importantes. En primer lugar, y refiriéndose a mi tierra natal, Navarra, como parte integrante de lo que él denomina ajustadamente País VascoNavarro, diferencia la distinta historia reciente, en este caso junto a Álava, por la común adhesión al Movimiento franquista en 1936 que trajo consigo el premio de un desarrollo económico a las dos provincias, acercándolas a las otras del país, Vizcaya y Guipúzcoa, más industrializadas desde mucho antes. Ese progreso precisamente había conllevado asimismo la secularización religiosa haciendo desaparecer el rural tradicionalismo carlista en esta parte de la Península, pues incluso el nuevo partido político del pretendiente Carlos Hugo, ya no tradicionalista, estaba casi totalmente secularizado. Sobre todo, este fenómeno de la secularización religiosa en la sociedad occidental y la laicidad de los estados democráticos contemporáneos también reflexionó Aranguren y de ello he dado cuenta como autor en un reciente libro (Aramburu, 2017).

El segundo supuesto consiste en que a ese desarrollo económico no correspondió un desarrollo cultural, por ceguera del Estado (o tal vez, en mi opinión, premeditadamente), hasta muy tardíamente con la creación de la Universidad (pública) del País Vasco. Para Aranguren parece no había sido significativa - y en ello opino no es del todo justo— la labor de la universidad eclesiástica de los jesuitas de Deusto ni el enclave del Opus Dei en Pamplona, este último sin incidencia alguna sobre la realidad nacionalista vasca, sin tener en cuenta la cátedra de Lengua y Cultura Vascas que se creó en esa universidad privada de Navarra y ocupó el gran patriarca de la cultura vasca José Miguel de Barandiarán en los años 1960. Sí reconoce, y en ello hace justicia, la irradiación religioso-cultural de los seminarios diocesanos, sobre todo del de Vitoria en la posguerra, en los que se han formado los hombres más preparados del país si bien, en contraste, se ha vivido la situación paradójica de aquella secularización religiosa creciente, aneja al desarrollo económico.

Asimismo, Aranguren alude justamente al papel en la política, manteniendo la llama nacionalista y en las costumbres locales, de los denominados curas vascos. Así, son bien conocidos los antecedentes eclesiásticos, de sacerdocio o seminario, de muchos de los líderes nacionalistas y de los de ETA. Menciona el caso de Telesforo Monzón, un viejo líder vasco que, como el italiano Toni Negri, unía en sí al teórico y al activista, y que repetía muchas veces que el abertzale había 


\section{MIKEL ARAMBURU ZUDAIRE}

de ser, a la vez, jelkide y gudari, que traducido del euskera es lo mismo que mitad monje y mitad soldado. Por tanto, la raíz funcional y estructuralmente religiosa de la banda terrorista y del partido que políticamente la ha representado, sin ser confesionales, es bien constatable. En resumen, para Aranguren cuatro han de ser los factores en el análisis del proyecto abertzale de comunidad nacional vasca: el juvenil, el nacionalista, el revolucionario-socialista y el apocalíptico-religioso (Aranguren, 1995, p. 683-84).

Otros dos puntos relacionados con todo lo que Aranguren va exponiendo creo que hoy serían aceptables. Primeramente, el paralelismo entre el terrorismo vasco y el irlandés, pues se han dado en dos países de arraigada tradición católica, un tanto arcaizantes culturalmente, desarrollados económicamente respecto a su entorno estatal (al menos la industrializada Irlanda del Norte) y donde sus jóvenes han vivido una cierta añoranza id́lica ante la saturación en el proceso vital de industrialización. Y el segundo punto, la conciencia de sí mismos que han tenido los miembros de ETA en un ambiente de sacralización de la violencia (micro) nacionalista que, según Aranguren, no pueden ser considerados santos, a lo sumo mártires de una causa sagrada, y que se creen héroes con todo el ingrediente narcisista que ello comporta, incluso hasta llegar a un narcisismo puritano, "tremenda y enajenadamente serio, con la enajenación del yo en el narcisista colectivo étnico" (1995, p. 685). Y añade algo que puede resultar chocante en principio pero que tiene su sentido si se piensa bien: con igual seguridad subjetiva podrían tomarse y ser macronacionalmente consideradas como heroicas las víctimas del terrorismo, pues he aquí el veneno de todas las guerras nacionales, las objetivas y las subjetivas, que además se viven secularizadamente a modo de guerras religiosas. Efectivamente, no pueden ser asimiladas a héroes stricto sensu en tanto que víctimas de una violencia ilegítima, ni tampoco condicionar la vida política democrática, ahora bien, sí merecen especial reconocimiento de la sociedad mediante la verdad, la justicia y la reparación del daño, como cualquier otra víctima de violencia ilegítima de distinto signo.

Por último, en los siguientes epígrafes, Aranguren aborda tanto las posibles salidas vascas del conflicto de Euskadi como las posibles opciones españolas (1995, p. 685-90). Sorprende un poco que, ya en aquellos lejanos 80, emplee ese lenguaje de dos partes bien separadas y equiparadas en interlocución, pues curiosamente todo lo que escribe a continuación sobre el tema vasco podría ser aplicado hoy, mutatis mutandis, al caso catalán, salvo que en este no han pasado 


\section{ESPAÑA, UNA MEDITACIÓN POLÍTICA: CATALUÑA Y EUSKADI}

antes por las oscuras décadas de violencias, sobre todo la terrorista de ETA pero también, sin ser equiparables, la de motivación política de grupos de extrema derecha o parapoliciales y organizaciones vinculadas al Estado o actuando en su nombre. Aranguren ve que el final del conflicto vasco iba a llegar y con él la hora de una nueva integración (diríamos ahora convivencia o reconciliación), y sorprende aún más que imagine un escenario hipotético de ruptura e independencia respecto a España como solución. Ello conllevaría, según su opinión y, en primer lugar, la disolución del frente existente entonces entre el Partido Nacionalista Vasco (PNV) y los revolucionarios ante el enemigo común y opresor español.

Como primera opción para ese escenario independiente plantea la hipótesis de un estado modelo comunista, pero le parece inviable de todo punto pues no sería reconocido en Occidente o se convertiría en una especie de isla de Cuba europea en brazos de la Unión Soviética. Hoy vemos casi con una sonrisa esta posibilidad tan lejana como imposible con todo lo que ha llovido después. $\mathrm{Y}$, sin embargo, muchas personas en el mundo seguimos buscando, tras el naufragio de las izquierdas, alguna alternativa real y eficaz para otro mundo posible que haga frente a los desmanes del neoliberalismo capitalista hegemónico. Entre esas personas no dudo en incluir al principal líder moral de la actualidad a nivel internacional, el papa Francisco, con su magisterio fiel al puro Evangelio y a lo mejor de la Doctrina Social de la Iglesia católica. Como segunda opción, Aranguren alude a un estado de socialismo tipo israelí o sionista, pero no dejaría de ser una contradicción con los términos mismos de un conflicto revolucionario marxista-leninista por el que parecía apostar ETA. En fin, una tercera opción imaginable de ruptura podría ser la comunitarista más tradicional, apegada al país (como la tecnología intermedia de Guipúzcoa) y con sus valores de retorno a la medida humana, a la naturaleza y a la antigua calidad de vida, a la religiosidad no dogmática (una de las raíces de movimiento vasco), a la descentralización y la autogestión, etc. Sin embargo, él percibe en ese momento que la juventud vasca no está por la labor de la ruptura total con la cultura establecida para regresar a la supuesta edad dorada de una arcadia bucólica como la predicada por el viejo carlismo. Quizá hoy en día la situación ha cambiado y el influjo, entre otras, de las corrientes ecologistas y altermundialistas con el deseo de una vida más simplificada, en una economía sostenible, abre las puertas a configurar otros modos de vivir y de organizar la sociedad. 


\section{MIKEL ARAMBURU ZUDAIRE}

Lo que vislumbra Aranguren, y en eso fue una vez más visionario o adelantado, es que la única salida vasca posible solo vendría a través del PNV cuya auténtica ideología, según él, como partido eminentemente burgués y moderadamente nacionalista que era, estaba a la derecha de la socialdemocracia. Yo pienso, en cambio, que su ideología ha ido evolucionando y de partido confesional en origen pasó a demócrata-cristiano con la guerra civil española de 1936 y hoy, desde los años setenta, se puede decir es humanista sin más adjetivos o a lo sumo social-cristiano, interclasista y bien enraizado en el país, con una praxis progresista en lo social y cultural pero más liberal en lo económico y por encima de todo pragmático ocupando la centralidad socioelectoral de Euskadi. En lo identitario me parece lo define muy bien la imagen del péndulo patriótico, con sus dos almas, una más autonomista y otra más independentista, en convivencia más o menos pacífica, según momentos, a lo largo de su más que centenaria historia (De Pablo, Mees y Rodríguez, 2001).

El partido jeltzale (JEL es el acrónimo del lema en euskera Jaun-Goikua eta Lagi-zaŕa o Dios y las Leyes Viejas, creado por Sabino Arana, fundador en 1895 del Euzko Alderdi Jeltzalea o Partido Nacionalista Vasco) sería, pues, el mayor beneficiario tanto en un estado de autonomía como de independencia, en cuyo caso Euskadi se convertiría en un país como Luxemburgo, que aún es más pequeño de extensión, dentro de la Comunidad Europea (el Estado español aún ni se había adherido). Así de fácil parece verlo en aquel momento Aranguren, aunque la juventud revolucionaria quedaría frustrada, incluso con la independencia y tras un alto coste de vidas humanas, como había quedado la española radical con la democracia suarista (del presidente Adolfo Suárez). No sé si ahora, con una independencia que no parece estar ni en la agenda a medio plazo de la mayoría del Parlamento o del Gobierno Vascos, se daría tal frustración, pues tampoco se sabe qué queda de la revolución o en qué consiste la ideología revolucionaria de la izquierda abertzale. Son, como se ha apuntado, otros tiempos, de redefinición ideológica y cambio acelerado en que no queda claro qué significa la soberanía nacional de un estado, y además tan pequeño, en un mundo globalizado e interdependiente, e incluso está en cuestión la entidad actual del mismo concepto de estado-nación.

Respecto a las salidas españolas, Aranguren muestra de nuevo su clarividencia de fino pensador y analista dando respuesta mediante opciones que hoy también nos resultan lógicas por muy irresponsables que parezcan políticamente. Así, la primera salida respondería a una reacción 


\section{ESPAÑA, UNA MEDITACIÓN POLÍTICA: CATALUÑA Y EUSKADI}

emocional o de desahogo apolítico, siempre a tener en consideración como reflejo de un estado de ánimo: que nos dejen en paz, eso sí, después de que pase al otro lado quien no quiera la independencia y se cierren las fronteras a cal y canto. Una segunda postura consistiría en forzar a los vascos a ser españoles al modo centralista, con declaración de estado de guerra, confrontación bélica y ocupación a sangre y fuego por las tropas del Ejército estatal. Bien se sabe que este "terror del Estado" (Aranguren, 1995, p. 688) solo exacerbaría el sentimiento nacionalista de todos los vascos sin solucionar de fondo el conflicto. Y la tercera salida, probablemente no más efectiva según Aranguren, supondría el restablecimiento de la pena de muerte para casos de terrorismo, pues la Constitución española de 1978 solo la contempla en tiempo de guerra. Pensemos por un momento que hoy de, al menos las dos primeras opciones (incluida la suspensión total o parcial de la autonomía por el excepcional artículo 155 de la Constitución), se está hablando desde altas instancias del Estado español y su Gobierno, llegada la situación extrema, para resolver lo de Cataluña y su proceso secesionista.

Con todo eso lo que Aranguren pretende llamar la atención es sobre algo también muy actual que él denomina juridicismo, es decir, utilizar una determinada interpretación, la suya, de la Constitución, entre quienes o no la votaron o lo hicieron a regañadientes y sin convicción y frente a una comunidad, la vasca, que mayoritariamente no la votó. Ciertamente está aludiendo también a la actual judicialización abusiva de la política, mal endémico de la democracia en España. Y aún añade Aranguren una cuestión más, adelantándose así a denunciar otro mal de alguno de nuestro dirigentes del presente aunque, al parecer, le produzca buenos réditos electorales: la voluntad de no hacer nada o noluntad, dejando que la situación degenere en completamente pourrie (se pudra) hasta el abandonismo tal como solía hacer el dictador Franco en los conflictos internacionales o "tendía a no-hacer el Gobierno de UCD (Unión de Centro Democrático, el partido del presidente Suárez), heredero legítimo, sin ruptura, de aquél” (Aranguren, 1995, p. 688).

Por otro lado, Aranguren recuerda, y me parece siempre muy necesario, que hay que considerar la responsabilidad histórica en todos estos temas. Durante la guerra civil del 36, el pueblo vasco - entonces sí con una opción, como se ha dicho, opuesta al navarro- fue aplastado en una contienda vivida por él como suya, diferente de la del Gobierno republicano de Madrid, y lo que se sembró entonces se cosechaba después e incluso ha llegado hasta el presente: "lo que 


\section{MIKEL ARAMBURU ZUDAIRE}

pasó, pasó, sigue pasando para nosotros y pesando sobre nosotros" (1995, p. 689). En cualquier caso, confiesa que se viven tiempos confusos y que le duele, y mucho, el terrorismo, pero le alarmaría igualmente:

que después de tantas víctimas suyas en impunidad empezaran a caer terroristas muertos por doquier...(o) un posible desmadre de la policía, vuelta repentinamente entusiasta de la Constitución, aunque no tanto, al parecer, de la supresión de la tortura. La eliminación del terrorismo no podrá lograrse sin la colaboración del pueblo vasco. $(1995$, p. 689)

Sin duda esta declaración resultaba muy valiente y polémica, y aún más hecha en aquella coyuntura, pero ha devenido providencial. Él defendía que no había otra opción practicable que buscar la solución política del conflicto y el interlocutor válido, insistía, era el PNV, eso sí, autodeterminado él mismo, dejando de estar a la zaga del radicalismo abertzale y cargando con el efectivo gobierno del País Vasco. En su opinión, al PNV no le interesaba la ruptura de la unidad nacional de España porque sus intereses se encontraban más amparados dentro de la comunidad hispánica que fuera de ella. No puede haber sido más atinado su diagnóstico y de hecho es lo que creo está ocurriendo hoy en Euskadi, afortunadamente terminada la lacra del terrorismo etarra, aunque con las heridas causadas todavía sin curar por completo.

De todos modos, su reflexión de intelectual iba más allá de la hora histórica y proponía emprender una tarea de desmitificación cultural del viejo concepto de nacionalismo. Por una parte, los nacionalistas vascos habrían de renunciar o posponer su sueño de una independencia nacional imposible y, por otra parte, los nacionalistas españoles, en un Estado cada vez más sometido y colonizado económicamente, deberían aferrarse menos a esa ficción de la unidad nacional, en realidad multinacional (plurinacional). Y afirmaba sin dudar: "La época de los nacionalismos toca a su fin, aunque él no sabía bien si en favor de grandes comunidades concretas, autónomas, democráticas y supranacionales, que sería lo deseable, o bien de grandes imperialismos, como era de temer" (Aranguren, 1995, p. 690). Considero que hoy todo es más complejo y si, por un lado, existe una legítima reivindicación nacional, que nunca ha desaparecido, como seña de identidad de los pueblos, por otro se han exacerbado nacionalismos de nuevo cuño fanáticos, totalitarios e imperialistas como el yihadismo, además de renacer ideologías populistas diversas, que están dominando a veces peligrosamente el escenario internacional.

Para cerrar el capítulo sobre Euskadi, Aranguren echa una mirada universal lo cual revela a las claras su talante - concepto tan querido por él y que explica magistralmente en su libro ya 


\section{ESPAÑA, UNA MEDITACIÓN POLÍTICA: CATALUÑA Y EUSKADI}

clásico de Ética, donde distingue bien el pâthos, lo que nos es dado por naturaleza o polo premoral, del êthos o carácter, adquirido por hábito, como polo moral (Aranguren, 1994)—, además de su pensamiento humanista y de cristiano heterodoxo. Para él, la organización probable del mundo, si era realmente democrática, había de ser supranacional. Pero la comunidad supranacional, europea $\mathrm{u}$ otra, pasaría necesariamente por las naciones en el sentido pre-nacionalista de la palabra, en tanto que una Europa de las patrias (expresión del presidente francés general De Gaulle) le resultaba una contradicción en los términos, y así manifestaba convencido:

A más o menos largo plazo se cobrará conciencia de esta obsolescencia del nacionalismo político. En cambio, la "nación", directamente arraigada en una cultura y una lengua -o manera de hablarla- propias, pero no estancadas sino en continuo renuevo, es la unidad cultural del porvenir. Y la revolución cultural es la que apunta a esas estructuras elementales de la vida cotidiana, de la vida de la "nación" con minúscula. (1995, p.690)

Tal vez, y en esta línea que propugna Aranguren, lo que hay que revisar es el concepto mismo de nación, la micro o con minúscula, que, sin negar su necesario sustrato cultural —no estrictamente étnico ni esencialista sino identitario transversal, y en su caso lingüístico propio-, lo combine con su articulación política, de modo creativo y democrático. Una nación más allá del estado-nación y sin fundamentalismos, para un nuevo mundo interrelacionado de estructuras supranacionales más fuertes como puede ser Europa, pero no meramente la de los mercados o la moneda común, sino también políticamente mejor cohesionada, equilibrada socialmente, de soberanía flexible y democracia compleja (Innerarity, 2017).

En fin, cabría también la revisión de otro concepto, el de España misma, para asumir de una vez su ser plurinacional (¿retomando y actualizando aquello de las Españas del tradicionalismo?), sin complejos ni prejuicios, con altura de miras y menos ideologización, y abierto a una particular y preferente vinculación supranacional, también política, con América Latina. Asimismo, estarían llamadas todas las naciones hispanas, las macro- y las micro-, de aquende y allende los mares, a formar otra gran y plural comunidad. Sin duda, un enorme y apasionante reto, intelectual y sociopolítico, que espera y merece nuevas meditaciones y acciones.

\section{Referencias}

Aramburu Zudaire, M. (2017). Un creyente entre los "éxtasis" del tiempo: heterodoxia, secularización y laicidad en el pensamiento de José Luis L. Aranguren. Madrid, España: Ápeiron Ediciones. 


\section{MIKEL ARAMBURU ZUDAIRE}

Aranguren, J. L. L. (1994). Ética. En F. Blázquez (Ed.), Obras Completas, vol.2 (pp.159-502) Madrid, España: Trotta.

Aranguren, J. L. L. (1995). España, una meditación política. En F. Blázquez (Ed.), Obras Completas, vol.3 (pp. 651-710). Madrid, España: Trotta.

De Pablo, S. Mees, L. Rodríguez, R. (2001). El péndulo patriótico: historia del Partido Nacionalista Vasco, 2 vols. Barcelona, España: Editorial Crítica.

Girard, R. (2006). La violencia y lo sagrado. Barcelona, España: Editorial Anagrama.

Innerarity, D. (2017). La democracia en Europa. Barcelona, España: Galaxia Gutenberg.

López de Cerain, R. (2016). Cuestiones varias. Burlada-Navarra, España: Sahats. 


\section{REVISTA STVLTIFERA DE HUMANIDADES Y CIENCIAS SOCIALES}

VOLUMEN 1, NÚMERO 1, PRIMER SEMESTRE DEL 2018

ISSN 0719-983X

\section{Artículos de Humanidades y Ciencias Sociales}

La violencia como espectáculo.

Juan Carlos Pérez Jiménez

El amo castrado.

Elisa Freijo Corbeira

España, una meditación política: Cataluña y Euskadi.

Mikel Aramburu Zudaire

Del saber sabio al saber dramatizado. Nuevos fundamentos para la aplicación permanente del teatro en el aula.

Luis Fernando Lara Coronado

Ontotecnia, ingeniería organizacional y actores emergentes.

Alejandro Ochoa Arias y Juan Antonio González de Requena Farré

\section{Reseñas}

Memoria Visual de Legua Emergencia, vida y oficio de Mario Alarcón.

Pedro Pablo Achondo Moya

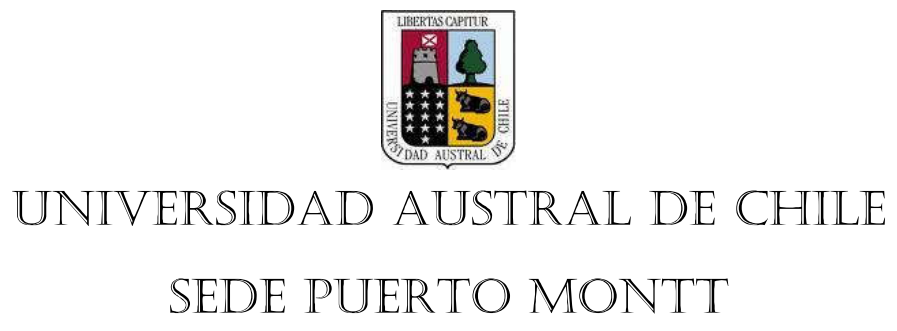

